\title{
Bioaccessibility of polyphenol and carotenoid from wild Irish berries subjected to an in vitro digestion procedure
}

\author{
A. M. O’Sullivan, Y. C. O'Callaghan, T. P. O’Connor and N. M. O’Brien \\ School of Food and Nutritional Sciences, University College Cork, Ireland
}

Berries are a relatively rich dietary source of fibre, vitamins, minerals and phytochemicals, which may have a wide range of human health benefits such as anti-cancer, anti-inflammatory and antioxidant activities ${ }^{(1)}$. Little information is available on the phytochemical content of edible wild Irish berries. In addition, the effect of digestion on the bioaccessibility of antioxidants and carotenoids has previously been examined in a wide range of foods; however, there is little available information relating to berries. Therefore, we measured the content and bioassessibility of phenols and carotenoids in hawthorn (Crataegus monogyna), whitebeam (Sorbus aria), rowan (Sorbus aucuparia), rosehip (Rosa super excelsa) and sloe (Prunus spinosa).

Berries were picked locally. For each berry, a $5 \mathrm{~g}$ sample was weighed, homogenised and subjected to in-vitro digestion ${ }^{(2)}$, which simulated human gastric and intestinal digestion. Ultracentrifugation was used to separate micelle fractions from the digested material. Total phenol content (TPC) of the undigested and micelle samples was measured using the Folin-Ciocalteu method. Both the undigested and micelle samples were saponified and carotenoids were extracted using hexane. HPLC was used to quantify the carotenoid content of the fruits. Bioaccessibility is defined as the amount of phenols or carotenoids transferred to micelles after digestion when compared with the original amount present in the food.

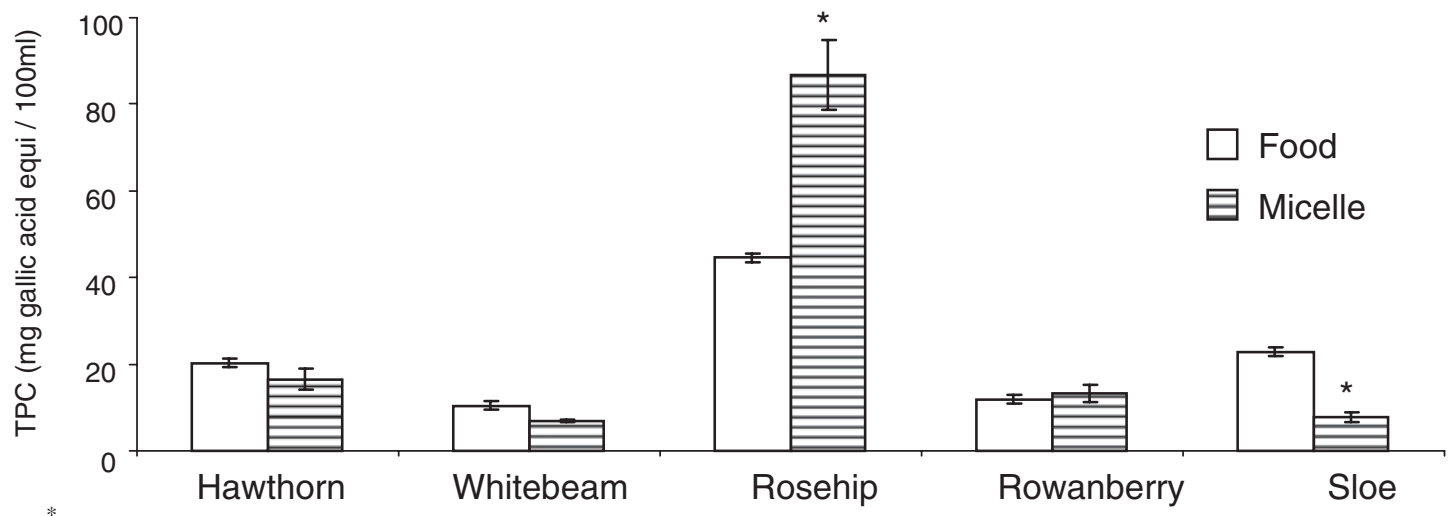

Denotes significant difference in total phenol content $(P<0.01) . n 4$ individual experiments. Statistical analysis was by repeated measures ANOVA followed by the Dunnett's test.

There was no significant difference between TPC in hawthorn, whitebeam and rowanberries before or after digestion. TPC of rosehip increased significantly $(P<0.05)$ in the micelle fraction, whereas TPC of sloe berries significantly $(P<0.05)$ decreased after digestion. $\beta$-Carotene was the predominant carotenoid found in whitebeam, rosehips and rowanberries ranging from 52 to $73 \mu \mathrm{g} / 100 \mathrm{~g}$ sample. In addition, whitebeam and rowanberries contained $\beta$-cryptoxanthin at concentrations of 10 and $35 \mu \mathrm{g} / 100 \mathrm{~g}$, respectively. Following digestion $\beta$-carotene levels ranged from approximately $1 \mu \mathrm{g} / 100 \mathrm{~g}$ in the micelle sample from the hawthorn to $6 \mu \mathrm{g} / 100 \mathrm{~g}$ in the micelle sample of rowanberry. Whitebeam and rowanberries contained 0.7 and $2.6 \mu \mathrm{g} \beta$-cryptoxanthin $/ 100 \mathrm{~g}$ in the micelle samples, respectively. In conclusion, bioaccessibility of phenols in berries is very high. Additionally, wild berries are a good source of certain carotenoids that are moderately bioaccessible and hence available for absorption by intestinal cells. 